\title{
Comparison of Observed Atmospheric Radio Refraction Effects With Values Predicted Through the Use of Surface Weather Observations
}

\begin{abstract}
B. R. Bean and G. D. Thayer
Contribution from Central Radio Propagation Laboratory, National Bureau of Standards, Boulder, Colo.

(November 9, 1962)

Past theoretical work has shown that it should be possible to estimate the atmospheric refraction of radio waves quite accurately simply from a knowledge of the radio refractive index at the surface of the earth. Prediction equations have been developed for use in estimating both elevation angle errors and radio range errors by means of performing linear regressions of ray-traced refraction variables on the values of surface refractivity, $N_{s}$, for a standard sample of radio refractivity profiles. In this paper the accuracy of these prediction equations is examined through a comparison with some precise measurements of total absolute refraction made with a radio sextant by the Collins Radio Company, some absolute elevation angle error measurements made at White Sands Missile Range in the lower atmosphere, and measurements of both relative elevation angle error and relative range and range difference errors made over various paths in the lower atmosphere. The validity of the refractive index profile sample used is confirmed through a test on some independent data obtained from four locations well outside the area of selection of the original sample. All results are shown to be consistent with the theoretical prediction model.
\end{abstract}

\section{Introduction}

The atmospheric radio refraction effects considered in this paper are of two general types: errors in measuring distance by means of timing the transit of radio signals between two points, known as radio range errors, and errors in estimating the elevation angle of a target by means of measuring the angle of arrival of radio signals from the target, known as elevation angle errors. Many methods have been proposed to take into account these refraction effects for the purpose of improving measurements by removing systematic bias. One of these involves the use of the surface value of the radio refractivity, $N_{s}$, a quantity which can be measured directly with a microwave refractometer, or calculated from the ordinary meteorological variables of temperature, pressure, and humidity, to predict values of either range error or elevation angle error; this method has been shown theoretically to be useful, with the accuracy increasing with increasing initial elevation angle [Bean and Cahoon, 1957; Bean, Cahoon, and Thayer, 1960; Thayer and Bean, in progress]. It is the purpose of the present note to compare recent experimental determinations of atmospheric refractive effects with values estimated theoretically from surface meteorological conditions.

\section{Theory}

The operation of a radio tracking system depends on the measurement, in some manner, of radio signals received from the target. The radio signals are transmitted in the form of radio waves which travel from the target to the tracking system. The form of these radio waves is distorted by the presence of the earth's atmosphere. Since solutions of the wave equation are extremely difficult to obtain for the case of general atmospheric propagation over a spherical earth, it is common practice to evaluate refraction effects by means of ray tracing, a process which is based on the use of Snell's law:

$$
\mu_{1} \sin \xi_{1}=\mu_{2} \sin \xi_{2},
$$

which gives the relationship between the angles of incidence $\xi_{1}, \xi_{2}$ of a plane wave front as it passes across a plane boundary between media of refractive index $\mu_{1}, \mu_{2}$.

It can be shown [Smart, 1931] that if the radio refractive index, $n$, of the atmosphere is a function only of height above a smooth spherical earth, then the application of Snell's law will result in the following ray-tracing equation, commonly referred to as Snell's law for a spherically stratified atmosphere:

$$
n r \cos \theta=n_{0} r_{0} \cos \theta_{0} .
$$

Here $n$, and hence $\theta$, the local elevation angle of any point on the ray path, are functions of $r$, which in turn is equal to the radius of the earth at the surface, usually taken as $r_{0}$, the point of origin of the ray path, plus the height, $h$, above the earth's surface. Figure 1 shows some of the geometry involved in ray tracing. 


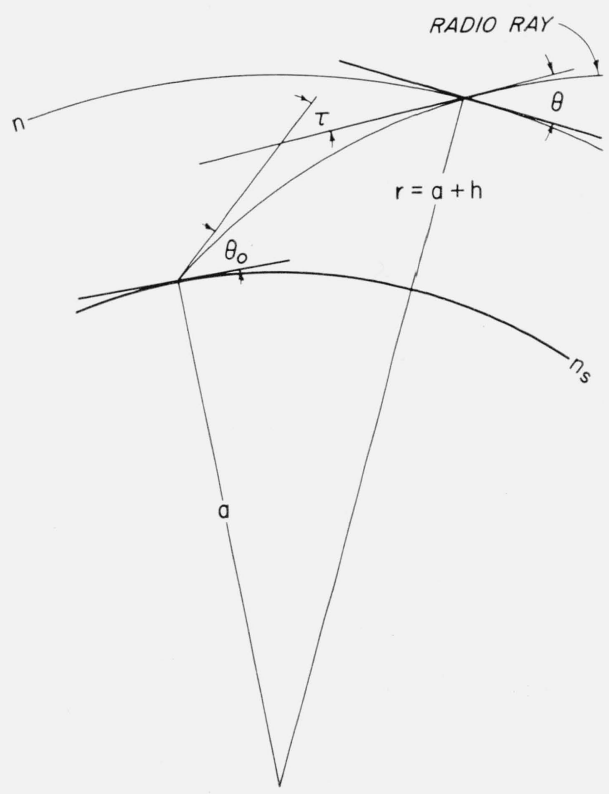

Figure 1. Geometry of radio ray refraction.

Based on the foregoing assumptions, the expression yielding the total refraction of the ray between the surface and any point, 1 , on the ray path can be derived as [ibid]:

$$
\tau=-\int_{0}^{1} \frac{d n}{n} \cot \theta
$$

the minus sign merely defines downward bending as positive.

One of the two types of refraction errors considered in this paper is the elevation angle error, $\epsilon$, which is the difference between the apparent direction to a target, as indicated by the angle of arrival of the radio wave front, and the true direction. This error is primarily a function of the refraction, or bending, of the radio ray, given by (3); for targets beyond the atmosphere the two quantities are asymptotically equal (with increasing range). The values of $\epsilon$ and $\tau$ at any point on the ray path obey the following inequality:

$$
\tau / 2 \leqq \epsilon \leqq \tau .
$$

Equation (3) can be integrated by parts, without knowledge of the refractive index profile, yielding

$$
\tau=\left(n_{o}-n_{1}\right) \cot \theta_{o}-\int_{0}^{1}(n-1) \csc ^{2} \theta d \theta
$$

or

$$
\tau=\left(N_{s}-N_{1}\right) \times 10^{-6} \cot \theta_{o}-10^{-6} \int_{0}^{1} N \csc ^{2} \theta d \theta
$$

where $N \equiv(n-1) \times 10^{6}$, the excess of the refractive index over unity in parts per million, and $N_{s}$ is the surface value of $N$. The subscripts 0 and 1 refer to values at the lower and upper parts on the ray path over which the integration is taken. It has been shown [Schulkin, 1952] that the integral part of (4) contributes less than 3.5 percent to the value of $\tau$ for $\theta_{0}$ larger than about $10^{\circ}$.

Thus the bending of a radio ray may be expressed by an equation of the form

$$
\tau=a+b N_{s}
$$

where $a$ and $b$ would be functions of the initial elevation angle of the ray, $\theta_{0}$, and the height (or range) along the ray path at which the bending is to be calculated. Such an assumption can be checked by examining the behavior of values of $\tau$, ray traced for a number of observed height-profiles of radio refractive index, plotted against the corresponding values of $N_{s}$. Such a plot is shown in figure 2, for a small initial elevation angle, $50 \mathrm{mr}$ (milliradians) (about $3^{\circ}$ ), and a "target" height beyond the atmosphere, $70 \mathrm{~km}$. The family of $N$-profiles used in ray tracing this sample of bending values is referred to as the CRPL Standard Sample. ${ }^{1}$ It can be seen from inspection of figure 2 that the assumption of linearity expressed in (5) is justified for this case. A similar conclusion can be reached from examination of data for other cases, including low target heights and elevation angles down to zero degrees, although for these extremes the degree of correlation between $\epsilon$ and $N_{s}$ is not as marked as that shown in figure 2 .

The other refraction variable treated in this paper is the radio range error, $\Delta R_{e}$, which is here defined as being that error incurred in measuring the distance between two points by means of timing the transit of radio signals between the points, and assuming that the velocity of propagation is equal to that of free space. For the case of a radio ray, this error is composed of two parts: the difference between the curved length of the ray path, called the geometric range,

${ }_{1}$ Meaning explained in section 3 .

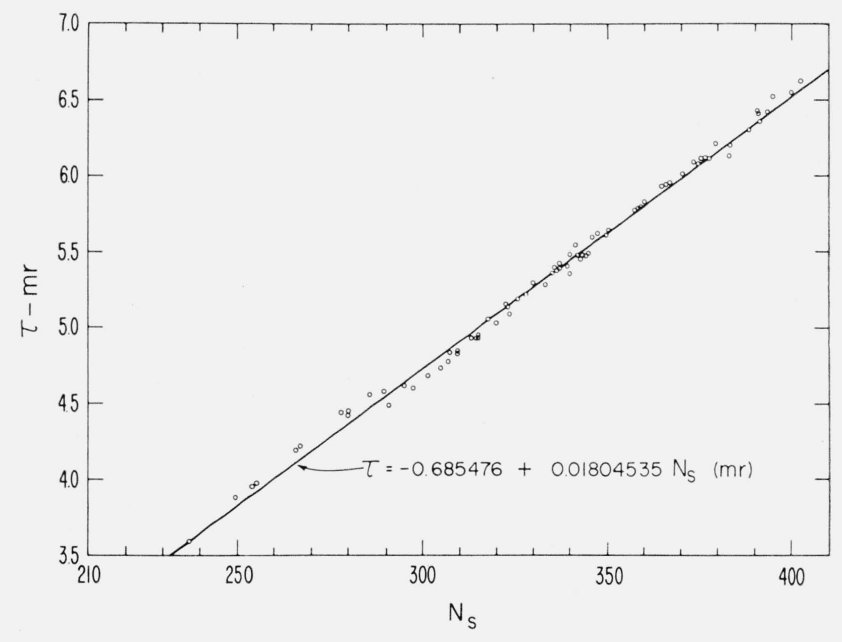

FIGURE 2. Total refraction at $\theta_{0}=50 \mathrm{mr}, \mathrm{h}=70 \mathrm{~km}$, for the CRPL Standard Sample.

Line indicates least squares linear regression of $\tau$ on $N_{s}$. 
$R_{g}$, and the true slant range, $R_{0}$; and the discrepancy caused by the lowered velocity of propagation in a refractive medium. The geometric range is given by

$$
R_{g}=\int_{0}^{h_{t}} \csc \theta d h
$$

and the apparent, or radio, range by

$$
R_{e}=\int_{0}^{h_{t}} n \csc \theta d h
$$

Thus the total radio range error, $\Delta R_{e}=R_{e}-R_{0}$, is given by

$$
\begin{aligned}
& \Delta R_{e}=\int_{0}^{h_{t}} n \csc \theta d h-R_{0} \\
& \Delta R_{e}:=10^{-6} \int_{0}^{h_{t}} N \csc \theta d h+\int_{0}^{h_{t}} \csc \theta d h-R_{0} .
\end{aligned}
$$$$
\text { or }
$$

The first term on the right-hand side of (6) is the "velocity" or "refractivity" error, $\Delta R_{N}$; the last two terms represent the geometric range error, $\Delta R_{g}$, which is the difference in length between the straight path, $R_{0}$, and the curved ray path, $R_{g}$. Table 1 gives some typical and extreme values of

\begin{tabular}{|c|c|c|c|c|c|c|c|}
\hline \multirow{2}{*}{$\theta_{3}$} & \multicolumn{3}{|c|}{ Typical $N_{s} \cong 320$} & \multicolumn{3}{|c|}{ Extreme: $N_{s} \simeq 400$} & \multirow{2}{*}{$\frac{\begin{array}{c}\text { Maxi- } \\
\text { mum }\end{array}}{\Delta R_{g} / \Delta R_{e}}$} \\
\hline & $\Delta R_{g}$ & $\Delta R_{N}$ & $\Delta R_{e}$ & $\Delta R_{g}$ & $\Delta R_{N}$ & $\Delta R_{e}$ & \\
\hline $\begin{array}{l}\quad \text { Milliradians } \\
0 \\
20 \\
50 \\
100 \\
200\end{array}$ & $\begin{array}{c}\text { Meters } \\
10 \\
2.5 \\
.7 \\
.14 \\
.02 \\
.001\end{array}$ & $\begin{array}{c}100 \\
62.5 \\
38.1 \\
22.26 \\
11.9 \\
5.01\end{array}$ & $\begin{array}{r}110 \\
65 \\
38.8 \\
22.4 \\
11.9 \\
5.01\end{array}$ & $\begin{array}{c}60 \\
4.5 \\
1.0 \\
.2 \\
.03 \\
.002\end{array}$ & $\begin{array}{r}165 \\
73 \\
43 \\
24.8 \\
13.0 \\
5.50\end{array}$ & $\begin{array}{l}225 \\
77.5 \\
44 \\
25 \\
13.0 \\
5.50\end{array}$ & $\begin{array}{l}\% \\
\sim 27 \\
\quad 6 \\
2.3 \\
.8 \\
.23 \\
.04\end{array}$ \\
\hline
\end{tabular}
range errors ray traced for observed $N$-profiles.
TABLE 1. Typical and extreme values of range errors for targets beyond the atmosphere

From table 1 it can be seen that the geometric range error, $\Delta R_{g}$, does not represent a significant proportion of the total range error except at very small initial elevation angles, between zero and about $3^{\circ}$. This being so, the behavior of the total range error will be primarily a function of the first integral in (6) for elevation angles greater than about $3^{\circ}$. The integral expression

$$
\Delta R_{e} \simeq 10^{-6} \int_{0}^{h} N \csc \theta d h,
$$

may be rewritten as

$$
\Delta R_{e} \simeq \csc \theta_{0} \times 10^{-6} \int_{0}^{h_{\iota}} \frac{N d h}{1-2 \sin ^{2}\left(\frac{\theta-\theta_{0}}{2}\right)+\cot \theta_{0} \sin \left(\theta-\theta_{0}\right)}
$$

or

$$
\Delta R_{e} \simeq \csc \theta_{0} \int_{0}^{h_{t}} N d h+\sum_{i=1}^{\infty}(-1)^{i+1} \int_{0}^{h_{t}} N\left[\cot \theta_{0} \sin \left(\theta-\theta_{0}\right)-2 \sin ^{2}\left(\frac{\theta-\theta_{0}}{2}\right)\right]^{i} d h,
$$

for $\theta<2 \theta_{0}, \theta_{0}>0$.

This expression is analogous to that derived for the case of ray bending, (4), and similarly the integral series on the right hand side of (7) contributes only 3 percent or less to the value of $\Delta R_{e}$ for $\theta_{0}$ larger than about $10^{\circ}$. From (7) one would thus suspect that the radio range error might be well estimated as a linear function of the integral of $N$ with respect to height. In treating this integral, it is informative to note that any given $N(h)$ profile may be "broken up" into three primary components:

$$
N(h)=N^{\prime}\left(N_{s}, h\right)+N^{\prime \prime}\left(h+h_{s}\right)+\delta N(h)
$$

where $N^{\prime}$ is that part of the profile which can best be expressed as a function of $N_{s}$ and height, $N^{\prime \prime}$ is a standard distribution of refractivity with respect to altitude above mean sea level $\left(h+h_{s}\right)$ which is independent of $N_{s}$, especially above the tropopause, and $\delta N$ represents a random component of the profile which cannot in general be accounted for a priori. The $N^{\prime}$ component is generally effective over the first few kilometers, while above 6 or $7 \mathrm{~km}$ altitude the $N^{\prime \prime}$ component forms the bulk of the profile [Bean and Thayer, 1959a]. Thus the integral of the $N$ profile with respect to height may be written as:

$$
\begin{aligned}
\int_{0}^{h_{\iota}} N d h=\int_{0}^{h_{t}} N^{\prime}\left(N_{s}, h\right) d h \\
\quad+\int_{h_{s}}^{h_{\iota}+h_{s}} N^{\prime \prime}\left(h+h_{s}\right) d h+\int_{0}^{h_{t}} \delta N(h) d h,
\end{aligned}
$$

or,

$$
\int_{0}^{h_{t}} N d h=F_{1}\left(N_{s}, h_{t}\right)+F_{2}\left(h_{s}, h_{t}\right)+\delta F\left(h_{t}\right)
$$

where $\delta F$ is the random contribution to the integral. For any particular $h_{\iota}$ then

$$
\int_{0}^{h_{t}} N d h=F_{1}\left(N_{s}\right)+F_{2}\left(h_{s}\right)+\delta F
$$


or

$$
\int_{0}^{h_{t}} N d h=F_{2}\left(h_{s}=0\right)+F_{1}\left(N_{s}\right)-F_{3}\left(h_{s}\right)+\delta F
$$

where $F_{3}=\int_{0}^{h_{s}} N^{\prime \prime}\left(h+h_{s}\right) d h$, and $F_{2}\left(h_{s}=0\right)$ is a constant.

It was found empirically, from integrated $N(h)$ profiles, that

$$
\int_{0}^{h_{t}} N d h \simeq a+b_{1} N_{s}-b_{2} h_{s} \pm \text { S.E. }
$$

the analogy between (8) and (9) is plain, where the standard error of estimate of (9), "S.E.," represents the standard deviation of $\delta F$ of $(8)$. The results of such an empirical study are shown in figure 3, for the CRPL Standard $N$-profile Sample, for $h_{t}$ beyond the atmosphere.

For any particular application of (9) at a single location the term $b_{2} h_{s}$ will be absorbed into the constant $a$, since $h_{s}$ does not vary. However the introduction of this term is necessary to explain the station elevation dependence of integrated $N(h)$ profiles when taken from a sample containing stations at widely differing elevations, such as the Standard Sample.

It is thus apparent that radio range errors, at least at the higher elevation angles, are primarily a linear function of $N_{s}$. That this is also true at comparatively low angles is shown in figure 4, for $\theta_{0}=50 \mathrm{mr}$ (about $3^{\circ}$ ) for the same profile sample. The reader should especially note the similarity of the distributions of the points about the regression lines between figure 3 and 4 , showing that the range errors at about $3^{\circ}$ are still primarily a function of the integral of $N$ with respect to height, or the range error at $90^{\circ}$.

It has thus been demonstrated that, theoretically, it should be possible to estimate both the angle of

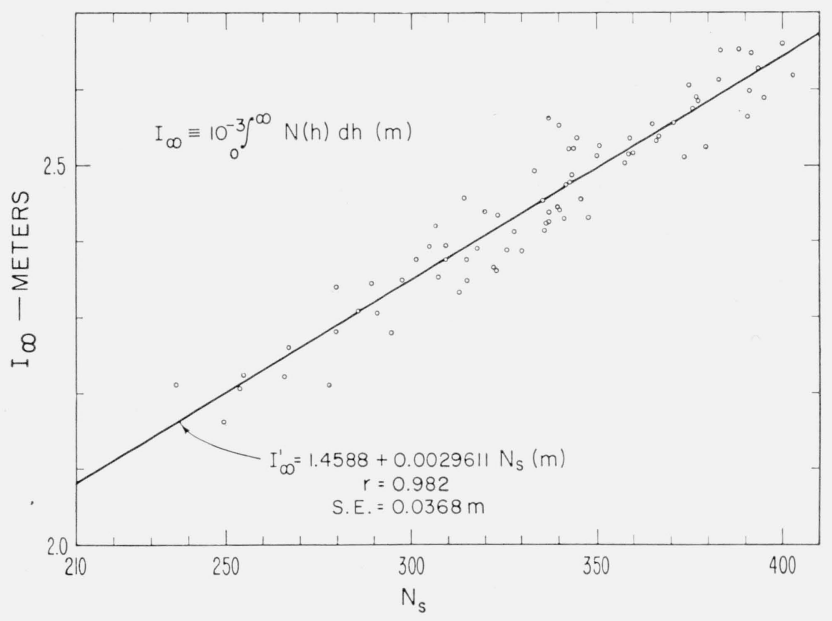

Figure 3. Integrated refractive index profiles for the CRPL Standard Sample.

The integral of $N(h)$ with respect to height is taken from the surface to $70 \mathrm{~km}$, above which point $N(h) \equiv 0$. The line represents the least squares regression of $I^{\prime}(\infty)$ on $N_{s}$, where $I^{\prime}(\infty)$ is the value of the integral as adjusted for the dependence on station (surface) elevation above mean sea level.

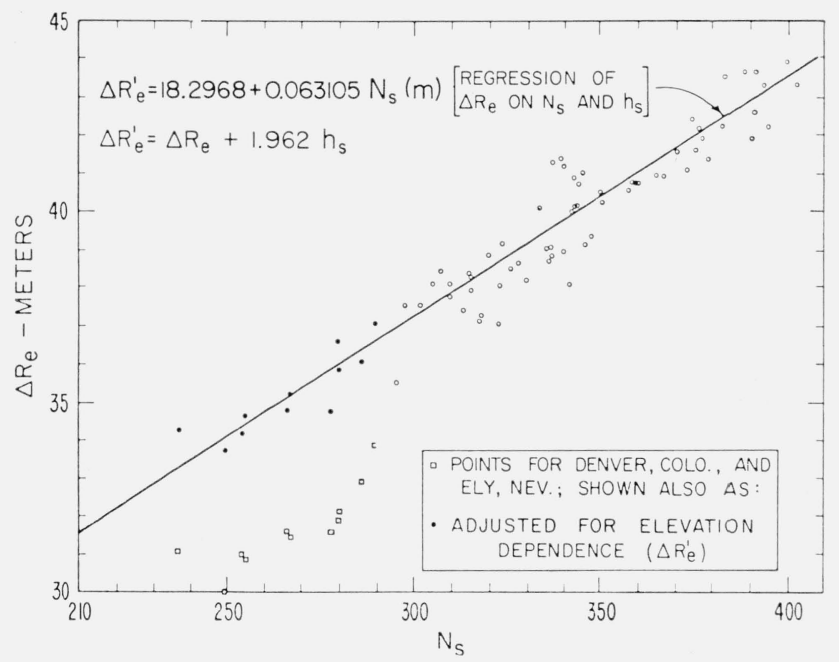

FIGURE 4. Total range error at $\theta_{0}=50 \mathrm{mr}, \mathrm{h}=\% 0 \mathrm{~km}$, for the CRPL Standard Sample.

The line indicates the least squares regression of $\Delta R_{e}{ }^{\prime}$ on $N_{s}$, where $\Delta R_{e}^{\prime}$ is the total range error as adjusted for the dependence on station elevation.

refraction of radio rays and errors in radio range measurements from measurement of the refractive index at the surface of the earth. This should be true for targets in or beyond the atmosphere, at elevation angles down to, and possibly lower than, $3^{\circ}$. In addition, if the behavior of refractive index profiles is similar in different parts of the world, it should be possible to specify "universal" values of the coefficients in (5) and (9), and to predict these values in advance by analysis of a large heterogeneous sample of refractive index profiles. In the succeeding sections of this paper it will be shown how this has been done, and a comparison will be made between the results so derived and the results of some measurements over actual radio paths.

\section{CRPL Standard Atmospheric Radio Refractive Index Profile Sample}

In the preceding section it was shown that, theoretically, it should be possible to estimate either radar elevation angle errors or radio range errors at any particular location by means of a system of linear equations in $N_{s}$, where the coefficients are functions of the target position. The target position can be specified by either the apparent elevation angle and target height, or the apparent range and target height (or as a third possibility, the apparent range and elevation angle), each having advantages in different situations [Thayer and Bean, in progress]. The equations recommended are:

$$
\epsilon=a_{1}\left(\theta_{0}, h_{t}\right)+b_{1}\left(\theta_{0}, h_{t}\right) N_{s} \pm \mathrm{S} . \mathrm{E}_{\cdot 1}\left(\theta_{0}, h_{t}\right)
$$

and

$$
\Delta R_{e}=a_{2}\left(R_{e}, h_{t}\right)+b_{2}\left(R_{e}, h_{t}\right) N_{s} \pm \mathrm{S} . \mathrm{E}_{.2}\left(R_{e}, h_{t}\right)
$$


where $\epsilon$ is the elevation angle error (see fig. 1), $\Delta R_{e}$ is the radio range error, $\theta_{0}$ is the apparent elevation angle, $h_{t}$ is the target height, $R_{e}$ is the apparent radio range, and S.E. is the standard error of estimate about the regression line of $\epsilon$ or $\Delta R_{\text {e }}$ on $N_{s}$. Values of the coefficients may be obtained by performing linear regressions of $\epsilon$ or $\Delta R_{e}$, as ray traced for an appropriate sample of radio refractive index profiles, upon $N_{s}$ for a large matrix of target positions. As a by-product of these calculations one also obtains, for each target position, a value of the residual error (the standard error of estimate) ${ }^{2}$ to be expected for the particular type of profile sample used.

In order to obtain a general set of equations to be useful under arbitrary conditions of location, climate, and weather, a large sample of $N$-profiles has been assembled which is believed to be representative of both mean climatic and geographic trends and the larger synoptic variations which may be encountered. This was done by choosing 13 radiosonde stations representative of the major geographic and climatic types of the world, and then choosing from each station $6 \mathrm{~N}$-profiles of particular types, two of which are typical of the extremes of monthly mean conditions for that location, and the other four of which are trpical of some of the variations which are found at that location [Bean, Cahoon, and Thaver, 1960]. The result is a sample of $77^{3} N$-profiles which has been found over a period of years to be a sound cross section of general refractive conditions and has thus been named the CRPL Standard Atmospheric Radio Refractive Index Profile Sample, hereafter referred to as the CRPL Standard Sample. Although the locations chosen for this sample are heavily weighted towards the United States, it has been found that the general behavior of the refractive index structure as inferred from the standard sample is typical of conditions experienced in most parts of the world [Misme, 1960].

The remainder of this paper will be devoted to some comparisons of observed radio refraction data with the predictions supplied by the CRPL Standard Sample, as derived from the linear regressions mentioned above.

Since the refraction measurements reported here consist of samples taken at particular locations over comparatively short periods of time, they should provide a test for the general set of coefficients derived from the Standard Sample; not only is the general theoretical approach tested against measured values, but the measurements coming from places of more or less homogeneous nature, they provide a check as to whether or not coefficients derived for a large heterogeneous sample of data are applicable also to individual places and times; i.e., they should reveal how much of the observed correlation of the heterogenous sample is derived from correlation between "classes" of data (in the statistical sense).

\footnotetext{
${ }^{2}$ For the Standard Sample the standard error of estimate is equal to the standard prediction error within \pm 1 percent over the range of $N_{s}$ from 200 to 470 , and will be used interchangeably with the latter.

3 One of the types could not be found for one of the stations used.
}

For a more thorough treatment of the CRPL Standard Sample and the associated regression coefficients for range error and elevation angle error, the reader is referred to a forthcoming publication [Bean, Thayer, et al., in progress].

\section{Comparison With Independent Data}

Before turning to an examination of the experimental refraction data and the degree of success realized in applying the theoretical prediction model to those data, it seems appropriate to examine the accuracy of the prediction model when applied to some independent theoretical (i.e., ray-traced) data. For this purpose four check stations were selected which were not only independent in the sense of not having been included in the original 13 station Standard Sample, but were from locations widely differing from the region of selection of the original sample. It was decided to select one station representative of an arctic type climate, one temperate, one tropical, and one from a "problem" climate area.

Amundsen-Scott station at the South Pole (lat. $\left.90^{\circ} \mathrm{S}\right)$ was chosen as the arctic type; this station was expected to present the most rigorous test of the prediction model (as based on the Standard Sample) that could be obtained anywhere in the world. In the first place the extreme arctic-continental climate, with almost no water-vapor contribution to the refractive index and the nearly incessant temperature inversion, is more alien to the Standard Sample than any other type; in the second place the station elevation is $2,800 \mathrm{~m}$, which is $900 \mathrm{~m}$ in excess of the highest station (Ely, Nevada, 1,908 m) included in the Standard Sample. These two effects were expected to augment each other as regards refraction.

Dakar, Senegal, on the western coast of Africa, was selected as a "problem" climate station; an inverse relationship exists there between $N_{s}$ and $\Delta N$ (the $N$-gradient over the first kilometer above the surface). A Congo basin station, Bangui, in what was French Equatorial Africa, was selected as the tropical location, and Moscow, U.S.S.R., was selected as the temperate location.

In order to combine brevity with comprehensiveness, ray tracings were done of the total refraction (bending at $70 \mathrm{~km}$ target height) at two elevation angles, $20 \mathrm{mr}$ and $100 \mathrm{mr}$, for six profiles from each location. The six profiles were selected as representing roughly the range of $N_{s}$ in winter (February), summer (August), and spring-fall (May and November), two profiles being selected from February, two from August, and one from each of May and November. The $20 \mathrm{mr}$ elevation angle was selected as representing roughly the lower limit of elevation angles for which the bending is expected to be strongly correlated with $N_{s}$ (say $r>0.9$ ), while at $100 \mathrm{mr}$ (about $6^{\circ}$ ) the correlation is expected to be extremely high (say $r>0.99$ ) and the refraction should be reasonably free of random profile effects.

The results of the ray tracings and the comparisons with predicted values are shown in figure 5. As expected, the results from the South Pole seem to depart significantly from the predicted values at 

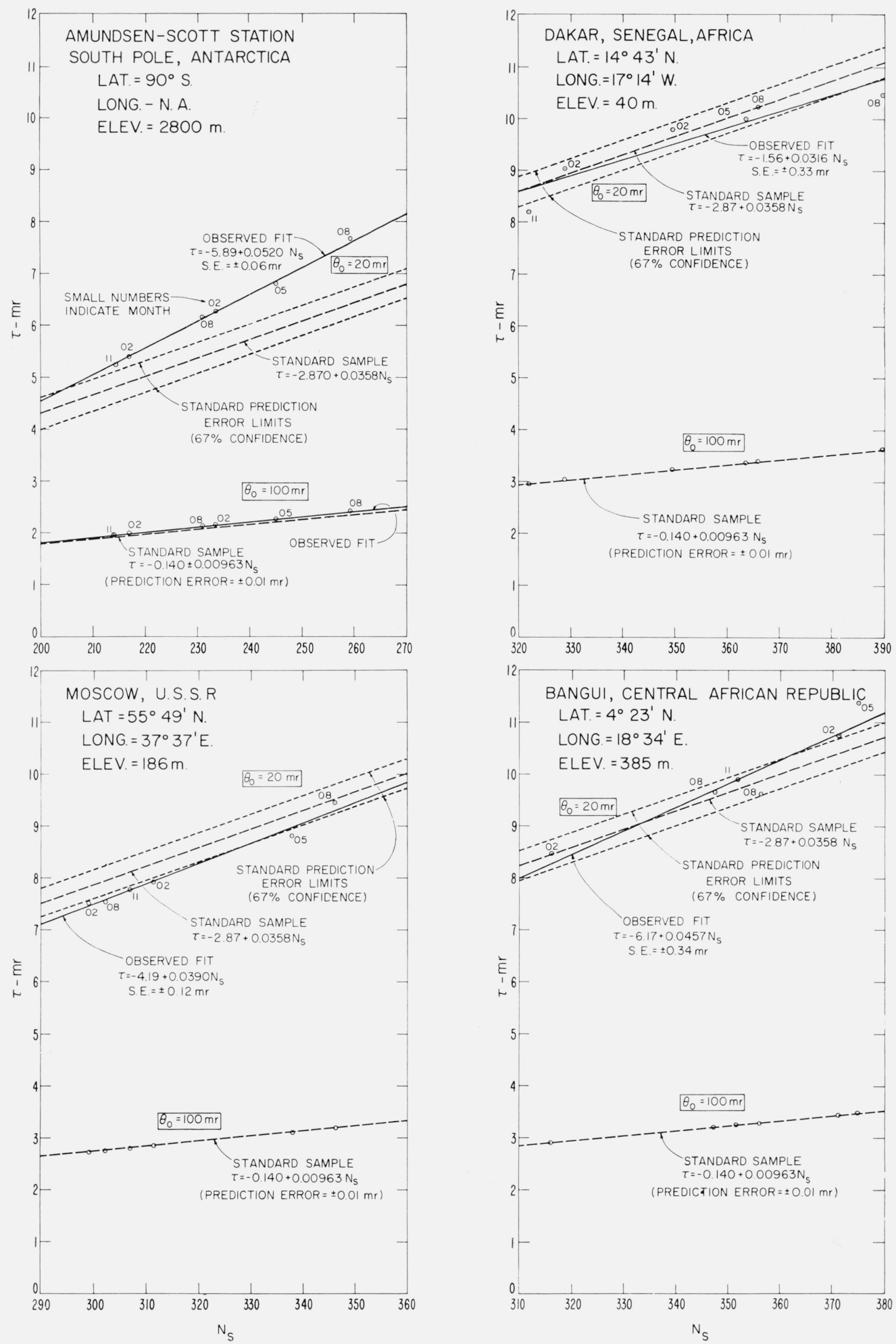

Figure 5. Comparison of predicted refraction at $\mathrm{h}=70 \mathrm{~km}$ for $\theta_{0}=20 \mathrm{mr}$ and $100 \mathrm{mr}$ from regressions of the CPRL Standard Sample and ray-traced values from four independent locations.

Solid lines indicate observed regressions of $\tau$ on $N_{s}$, dashed lines indicate regression lines from the CRPL Standard Sample and their standard prediction errors ( $67 \%$ confidence limits). Prediction error limits not shown for $\theta_{0}=100 \mathrm{mr}$ because of the very small difference from the regression lines. 
least for the $20 \mathrm{mr}$ elevation angle. At the $100 \mathrm{mr}$ elevation angle some of the calculated points lie more than one standard deviation from the predicted line (the theoretical prediction error is too small to show on the graph clearly), however, in all four cases the differences are less than 50 microradians, a figure which as shall be seen may represent the limit of accuracy obtainable from the atmosphere in actual practice. At angles over $100 \mathrm{mr}$ the errors would be smaller; in fact they should tend to decrease in inverse proportion to the square of the initial elevation angle, as indeed they do between 20 and $100 \mathrm{mr}$.

A conclusion which may be drawn from the above results is that any regions where the prediction model based on the Standard Sample would not be expected to provide the theoretical accuracy are probably regions of climatic extremes, and at least for the case of angular errors the effects will be negligible for elevation angles of a few degrees or more. As an interesting aside it can be noted that apparently the Antarctic may be a desirable area for tracking systems location, at least with respect to atmospheric refraction effects, since (most likely because of the lack of substantial water vapor and the relatively homogeneous conditions) the prediction error for $\theta_{0}=20 \mathrm{mr}$ in figure $5 \mathrm{a}$ is only about one-fifth as large as for temperate climates, indicating a possibly more stable atmosphere (even $90 \%$ confidence limits for the S.E. in figure 5 a vields a value less than half of the theoretical temperate value of $\pm 0.286 \mathrm{mr}$ ).

\section{Comparison With Experimental Results}

Before comparing the theoretical and experimental results it is appropriate at this point to examine what one would expect to observe on the basis of propagation theory. In the case of angular errors it is expected that propagation through the real, turbulent atmosphere will produce random variations in the shape of the incoming wavefront, so that measurements made with systems in which the receiving antenna is alined with the incoming signal will have random variations introduced in addition to the ordinary refraction effects. Since these variations will probably not be a function of elevation angle to any great extent, this implies that the residual variance in predicting the elevation angle errors will probably always be greater than predicted from theoretical (static) considerations, and that there will probably be some minimum value of this variance for very large elevation angles. Thus in some cases the residual errors will probably not decrease steadily with increasing elevation angle, but will tend to flatten out at some point and assume a more or less constant value above that point. These effects will be complicated in comparing one set of data with another by such things as differences in the location or time of day or season in which data is taken, and instrumental effects such as aperture averaging.

The case of range errors is more straightforward. The effects of turbulent atmospheric inhomogeneities are expected to average out over regions of abnormally high or low density, or water vapor concentration, when considering the transit time of particular points on the wave front. Hence the effect on the residual range errors is expected to be small, and the observed values are expected to compare rather well with the predicted (theoretical) values.

Turning first to the comparison of observed and predicted elevation angle errors, figure 6 shows some data on the mean refraction of $1.85 \mathrm{~cm}$ radio waves received from the sun, a target at essentially infinite range so that the elevation angle error is identical with the total angular bending of the radio ray, $\tau$. The data shown in figure 6 were obtained by tracking the sun with a precise radio sextant developed by the Collins Radio Company, and were collected in August through December of 1959 at Cedar Rapids, Iowa [Anway, 1961]. These data represent essentially instantaneous measurements. The mean of all observations at each elevation angle is plotted for elevation angles ranging from $2^{\circ}$ to $65^{\circ}$, and the mean value of $N_{s}$ associated with each point is about 332 ; the curve for the mean bending of the CRPL Standard Sample corresponds to the mean value of $N_{s}$ of 334.6 for that sample and hence the data should be comparable. The standard deviation "wings" refer to the standard deviation of the individual "instantaneous" data, not to the standard error of estimate of the mean value. The close agreement observed for elevation angles between $2^{\circ}$ and $35^{\circ}$ constitutes not only a confirmation of the usefulness of the Standard Sample, but also a verification of the accuracy of ray-tracing theory

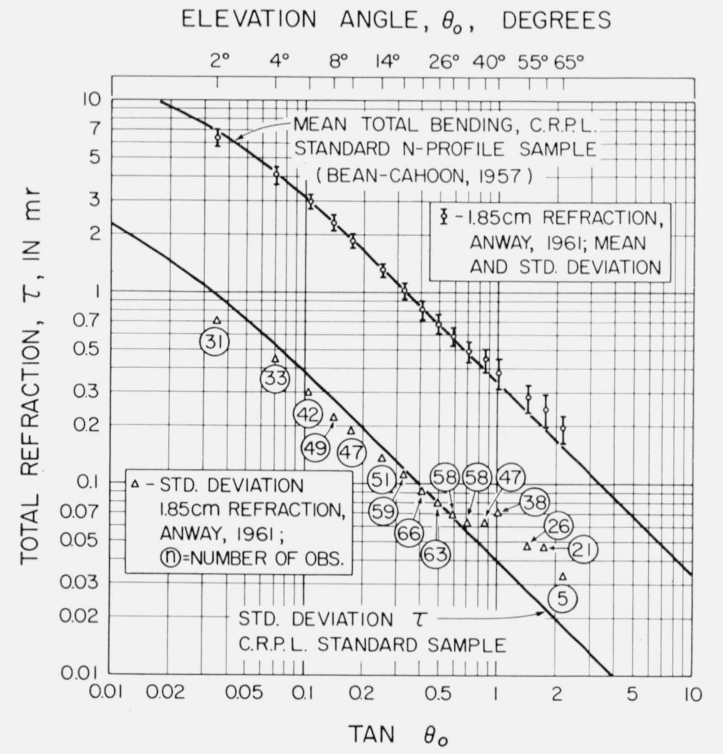

Figure 6. Comparison of measured total atmospheric refraction of $1.85 \mathrm{~cm}$ radio waves at Cedar Rapids, Iowa, with values predicted from $\mathrm{N}_{\mathrm{s}}$.

Upper curve and data points: mean value of refraction; lower curve and data points: the standard deviation of the refraction values about the mean. The wings on the mean refraction data points indicate the limits of the standard deviation of the observed data. 
in estimating radio wave refraction in the actual, and thus heterogeneous, atmosphere. The standard deviation of the Collins data (shown on the lower part of fig. 6) is generally lower than for the standard sample, but this is to be expected in view of the larger range of climatic variation contained in the CRPL standard profile sample. The apparent discrepancies in the measurements made at elevation angles over $40^{\circ}$ are apparently due to some slight inaccuracies in the calibration procedure used on the radio sextant during the period of data acquisition [Marner, private communication]. ${ }^{4}$ In fact, the data shown in figure 6 are almost precisely what one would expect to observe if all of the measured values of refraction were increased by a systematic calibration error of about 50 microradians over their correct values. The standard deviations in figure 6 tend to flatten out at high elevation angles, an effect which is to be expected theoretically as pointed out previously. At any rate, the largest difference between the observed data and the predicted curve in figure 6 , at elevation angles over $30^{\circ}$, is only about 50 microradians or $10 \mathrm{sec}$ of are (the angular diameter of the planet Mars at its average distance from the earth is $10^{\prime \prime}$, an angle not discernible to the naked eye). Although this discrepancy might be significant militarily, it is only about $1 / 2$ percent of the diameter of the target sun and is probably near the limit of accuracy of the equipment used.

Figure 7 shows the results of the specific measurements reported by Anway for the radio sextant for all cases at an elevation angle of $8 \pm 0.09^{\circ}$; each point represents an "instantaneous" reading. The solid line represents the linear regression of the measured refraction data on the values of $N_{s}$; the dashed line shows the predicted linear relationship derived from least squares fits to the CRPL standard sample raytraced refraction data. The mean bias between the

${ }_{4}$ The data for the highest elevation angles in figure 6 were necessarily collected during the early part of the period when the sun was higher in the sky. In a private communication, Anway states that the mean $N_{s}$ applicable to the data at $60^{\circ}$ to $65^{\circ}$ was 358 rather than 332 ; this difference would account for about at $60^{\circ}$ to $65^{\circ}$ was 358 rather than 332 ; this difference would account for about
one-third of the discrepancies noted, reducing the residual bias to a maximum of about 40 microradians.

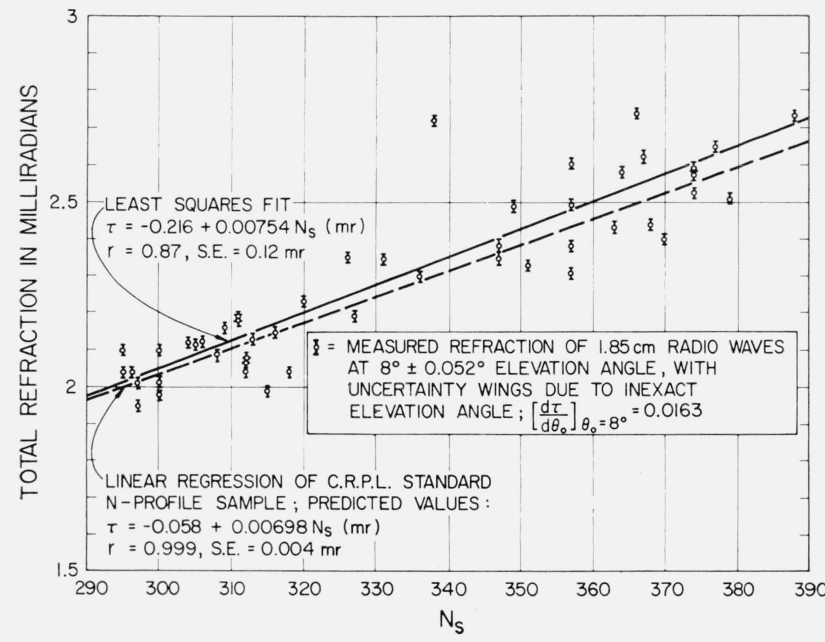

Figure 7. $1.85 \mathrm{~cm}$ radio refraction at an elevation angle of 8 deg, Anway, 1961, Cedar Rapids, Iowa. two lines is about 40 microradians, interestingly close to, and in the same direction as, the apparent calibration error noted in the mean refraction data at high elevation angles. The standard error of estimate is considerably higher than predicted; however, the rms uncertainty of $\pm 0.052^{\circ}$, or \pm 0.91 $\mathrm{mr}$, in the apparent elevation angle would be sufficient by itself to increase the standard error of estimate to about $\pm 0.017 \mathrm{mr}$, which is four times larger than the predicted value. It is not known how much of the total standard error of $\pm 0.12 \mathrm{mr}$ is due to measurement errors as opposed to unforeseen fluctuations in actual atmospheric refraction.

Figure 8 shows some results of measurements taken at Cape Canaveral, Fla., on November 1-3, 1959 [Janes and Thompson, to be published] at a very low elevation angle, about $0.7 \mathrm{mr}$ or $0.04^{\circ}$. These are "instantaneous" measurements, taken at halfhourly intervals, of the phase difference fluctuations between the signals from a beacon as they arrived at the upper and lower terminals of a vertical $24-\mathrm{ft}$ baseline, thus being very closely equivalent to a measurement of the fluctuations in the angle of arrival of the wave front at the centerpoint of the baseline (the altitude difference between this point and the target beacon is referred to as the "mean" target height). Since ouly the fluctuations and not the total phase differences were measured, only the slope and scatter of the elevation angle errors as a function of the observed $N_{s}$ data can be compared with the predicted values from the CRPL standard sample. The zero point on the graph is set by the predicted mean value for the sample. The correlation coefficient is, as expected, only 0.57. In this case the scatter of the observed data is well inside the limits of the standard error of estimate of the regression for the standard sample, even at this very small elevation angle where horizontal changes in the $N$ profile can exert a large effect on elevation angle errors.

Figure 9 shows the results of a comparison between predictions of elevation angle errors estimated from the CRPL standard sample and some measurements

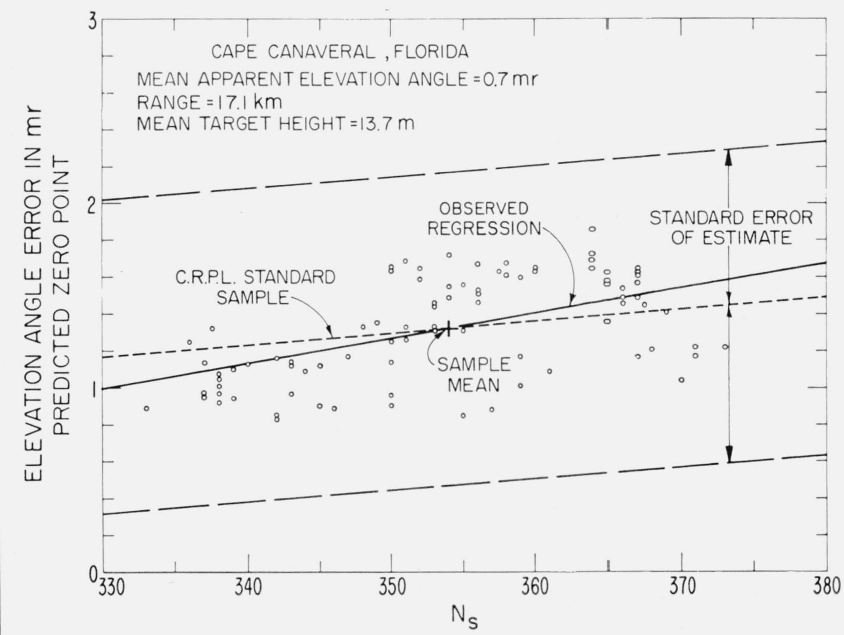

Figure 8. Elevation angle fuctuations from phase differences taken across a 24-ft vertical baseline, at Cape Canaveral, Fla. 
made with a $6 \mathrm{~cm}$ radar at Tularosa Basin, N. Mex., [Anderson, Byers, and Rainey, 1960]. Each point represents the mean of five "instantaneous" readings made at 1 -min intervals over a period of $4 \mathrm{~min}$. The standard deviation of each five-reading group averaged $0.16 \mathrm{mr}$, and the maximum range in any one group was $0.58 \mathrm{mr}$. The radio energy was propagated over a 45 -mile path at a mean apparent elevation angle of $18 \mathrm{mr}$; the target was a beacon located on a mountain peak 5610.5 feet higher than the desert floor where the radar was located. The data in figure 4 show that even for this rather extreme case, where the degree of correlation between $N_{s}$ and $\epsilon$ is expected to be only 0.4 , agreement is obtained between:

(1) The predicted and observed mean refraction,

(2) the observed and predicted slopes of the $\epsilon$ versus $N_{s}$ relation,

(3) the observed and predicted residual errors of predicting $\epsilon$ from $N_{s}$ alone.

The small discrepancy between the intercepts (i.e., between the mean refraction) of the observed and predicted $\epsilon$ versus $N_{s}$ lines may be perhaps attributed to, for example, antenna lobe pattern distortion caused by differential refraction, or defocusing [Wilkerson, 1962].

The remaining data which are examined were of necessity taken in such a manner as to have a rather high degree of autocorrelation (trends). Such data are not as suitable for confirming the accuracy of a regression prediction process as are independent data. A discussion of this is included at the end of the paper.

Turning to examination of radio range errors, figure 10 shows the results of some measurements of apparent radio range fluctuations over a $25-\mathrm{km}$ path on the island of Maui, Hawaii, on November 9-11, 1956 [Norton et al., 1961]. These measurements were made at 1-hr intervals, and are essentially "instantaneous" values. The target beacon was situated on the summit of Mount Haleakala at an

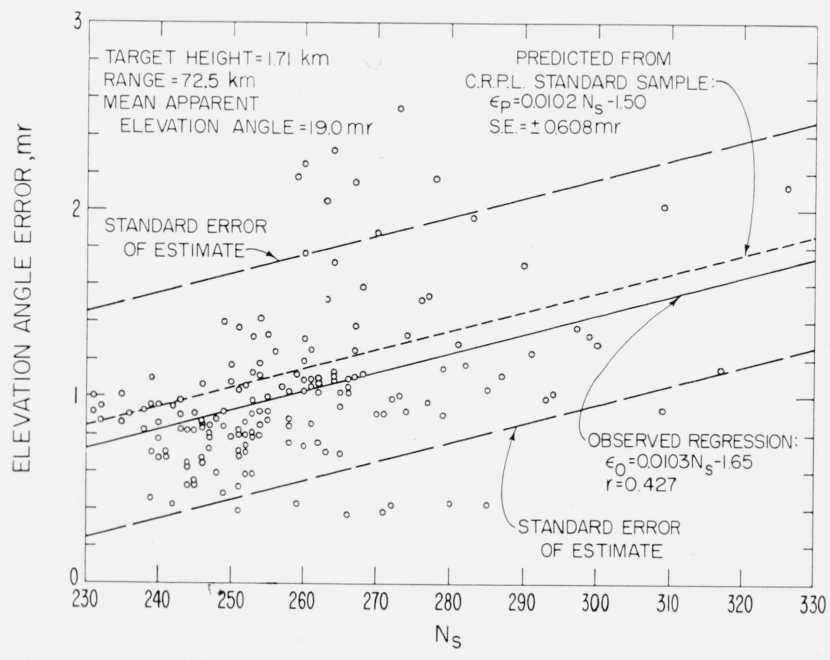

Figure 9. Measured refraction of C-band radar at Tularosa Basin, New Mexico. elevation of $10,025 \mathrm{ft}$, while the "ground" station was near Puunene Airport at an elevation of $104 \mathrm{ft}$, thus yielding a target height of $3.046 \mathrm{~km}$, in a region of critical target heights for prediction of radio range errors in tropical climates [Thayer and Bean, in progress]. The measured range fluctuations (absolute errors not measured) are plotted against values of $N_{s}$ taken at about the same time (mostly 15 to 20 min later) by U.S. Weather Bureau personnel at the Puunene Airport weather station. The agreement between observation and prediction is fairly good, especially when one considers that only 32 of the 86 points lie outside of the predicted standard error of estimate limits, while chance would indicate that 29 points would exceed these limits. Also, it should be kept in mind that in this case, as for all except the Collins data, the target beacon is located on the surface of the earth, whereas the predictions from the CRPL standard profile sample are derived for targets in the free atmosphere; there is undoubtedly some bias introduced in this way.

As a part of a continuing investigation into the atmospheric limitations imposed on electronic distance measuring equipment, some measurements have been made recently by the Lower Atmosphere Physics Section, NBS, of both range errors and range difference errors (across a phase-differencing baseline) over a propagation path near Boulder, Colo. Figures 11 and 12 are based on some of the preliminary results of these measurements [Thompson, 1962]. Figure 11 shows the results of measurements of the fluctuations in apparent range, made at half-hour intervals on May 9-11, 1961, over a $15.5 \mathrm{~km}$ path between a transmitting beacon on Green Mountain at an elevation of $2242 \mathrm{~m}$ and a receiving antenna located near Boulder Creek at an elevation of $1554 \mathrm{~m}$, the true target height thus being $688 \mathrm{~m}$. The apparent range fluctuations, expressed in parts-per-million of the $15.5 \mathrm{~km}$ path length (with an arbitrary zero since the total range was not measured), are plotted as a function of the surface value of the refractive index taken at a point quite close to the lower terminal. Quite good

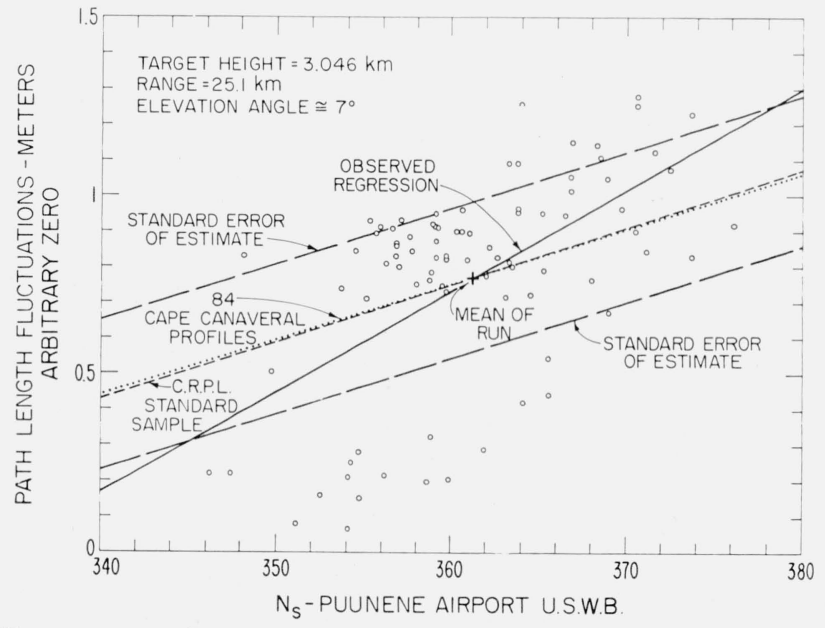

Figure 10. Range error fluctuations observed on Maui path. 


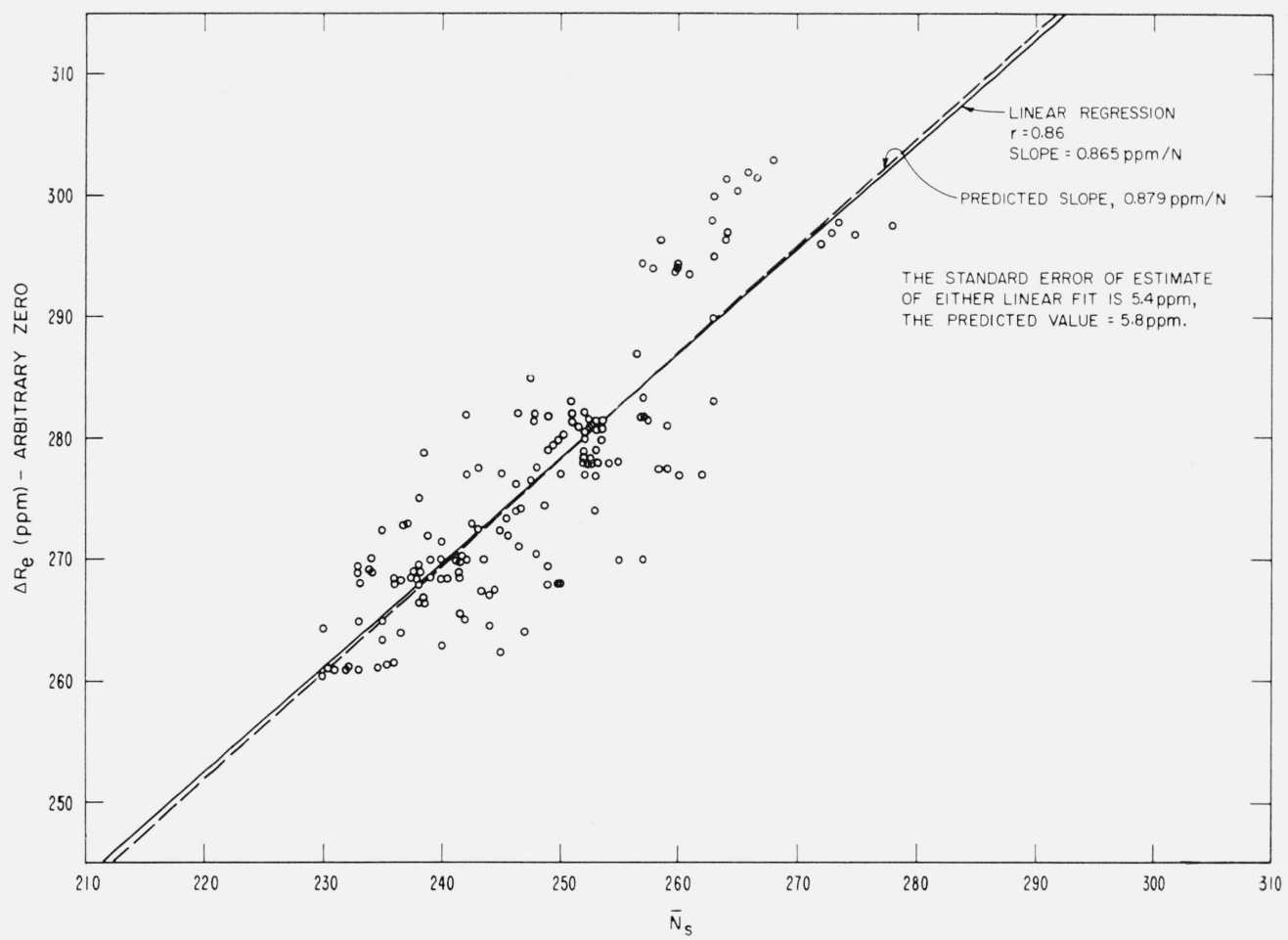

Figu RE 11. Range error fluctuations observed over the Boulder Creek, Green Mountain path, Colo.

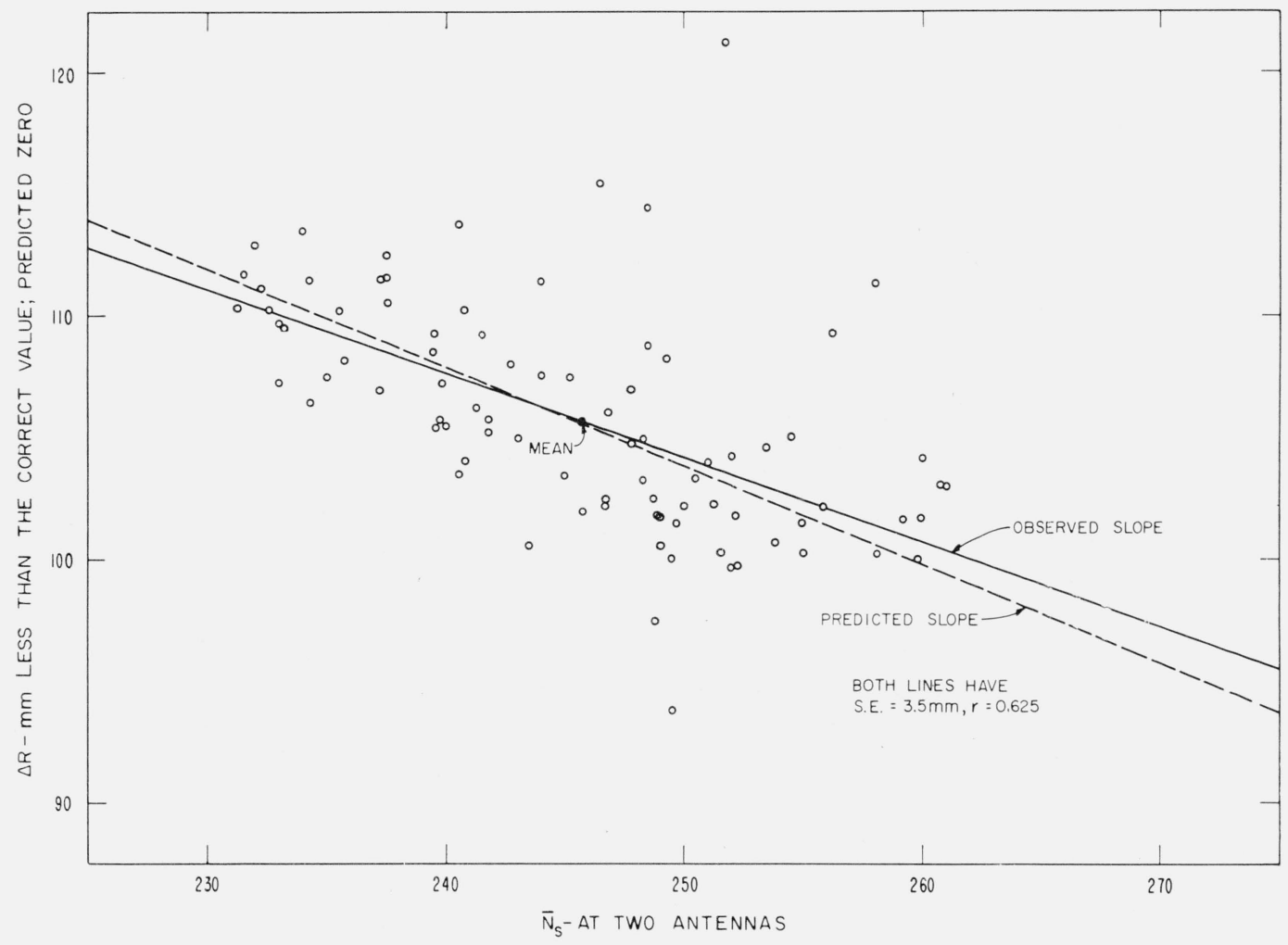

FiguRe 12. Range difference fluctuations observed over a 460-m in-line baseline, Boulder Creek, Green Mountain path, Colo. 
agreement is seen between the simple linear regression of the observed $\Delta R_{e}$ values on $N_{s}$ and the predicted linear relationship obtained from the CRPL standard sample. Note that both lines have statistically equal standard errors of estimate with respect to the observed data.

Figure 12 shows the results of the range difference measurements made over a $460-\mathrm{m}$ baseline essentially in line with the transmission path, where the second antenna was farther from the target beacon than the primary antenna. Here the range difference fluctuations (again with an arbitrary zero) have been plotted as a function of the mean value of $N_{s}$ measured at each end of the baseline. The zero point on the graph is set by the predicted mean of the sample. In this case there seems to be some discrepancy between the regression of the data and the predicted slope, however, note that the standard errors of estimate for the two lines are, to two significant figures, equal, indicating that the difference in the slopes is probably statistically insignificant.

There are some data points in figure 12 having a rather large deviation from the regression lines. Statistical theory (using the "Student" $t$-distribution for 84 degrees of freedom) shows that, if the data points are drawn from a normally distributed population, there should be only one point having a deviation of more than $\pm 9 \mathrm{~mm}$ from the observed regression line. There are in fact five such points in figure 12 , four above and one below the line. If these five points are "thrown out," on the grounds that they weight too heavily the extremes of the distribution of data points (this is especially true when using least squares regression), and the regression is then redone using the remaining 81 data points, the resulting value of the slope is $-0.385 \mathrm{~mm} / N$-unit, with $r=0.77$, compared to the predicted slope of $-0.381 \mathrm{~mm} / N$ unit, a rather close agreement.

\section{Discussion of Results}

As a summary of the results of the experimental versus theoretical comparisons given in the preceding section, a statistical analysis has been run on the significance of the differences between the slopes of the observed and predicted regression lines. In order to make the tests more stringent, it was assumed that the slopes derived from the Standard Sample should be taken to be the slopes of the population regression lines $(\beta)$, thus yielding an estimate of the significance of the departure of the observed slope from the assumed population value.

A value of $t$ was first calculated for each case using the relation [Bennett and Franklin, 1954]

$$
t_{j-2}=\frac{\left|b-\beta_{0}\right| \sqrt{\Sigma\left(x_{i}-\bar{x}\right)^{2}}}{\text { S.E. }},
$$

where $b$ is the observed slope, $\beta_{0}$ the assumed population, or theoretical, slope, $x$ refers to the independent variable in each regression, $N_{s}$, S.E. is the standard error of estimate, and $t_{j-2}$ is the value of $t$ for ${ }_{j-2}$ degrees of freedom. From $t_{j-2}$ confidence limits for $\beta$ at the $100(1-\alpha)$ percent level can be calculated from [ibid]

$$
b-\frac{t_{j-2}, \alpha^{\mathrm{S} . \mathrm{E}} .}{\sqrt{\Sigma\left(x_{i}-\bar{x}\right)^{2}}}<\beta<b+\frac{t_{j-2}, \alpha^{\mathrm{S} . \mathrm{E}}}{\sqrt{\Sigma\left(x_{i}-\bar{x}\right)^{2}}}
$$

The probability that the observed value $b$ would have fallen outside of these limits by chance is $\alpha$. Many statisticians consider a value of $t_{j-2}$ falling below the $100 \alpha=5$ percent level to be not significant, between the 5 percent and 1 percent levels to be of questionable significance, and over the 1 percent level to be significant [ibid]. An observed slope $b$ falling

$$
\left|b-\beta_{0}\right|>\frac{t_{j-2}, 0.01^{\text {S.E. }}}{\sqrt{\Sigma\left(x_{i}-\bar{x}\right)^{2}}},
$$

would thus be taken to represent a significant departure from the value $\beta_{0}$, and would thus imply the possibilities

(a) $\beta_{0}$ does not represent $\beta$ or

(b) $b$ represents the regression of data from a population different than that used in determining $\beta_{0}$, or

(c) both.

Before making the significance tests, however, the value of $j$, the number of independent observations going into the determination of $b$, must be known. In general, data of the type presented here are more or less highly autocorrelated, and hence not all independent. The data presented here, with the possible exception of the Collins data and the Tularosa Basin data for which the calculations could not be performed, have autocorrelation coefficients $r_{k}$, for lag $k(k=1,2,3$ units of time between successive measurements) that can be approximately described by

$$
r_{k} \simeq\left(r^{\prime}\right)^{k}
$$

and for this type of data the effective number of pieces of independent data, $j$, is given by [Brooks and Carruthers, 1953]

$$
j=n\left[\frac{1-r^{\prime}}{1+r^{\prime}}\right] .
$$

For the data treated here weighted mean values of $r^{\prime}$ were calculated from

$$
r^{\prime} \cong \frac{r_{1}+4 r_{2}^{1 / 2}+9 r_{3}^{1 / 3}+\ldots k^{2} r_{k}^{1 / k}}{1+4+9+\ldots k^{2}}
$$

where $k$ was the largest lag for which the autocorrelation coefficient was calculated, usually 4 or 5 . No special justification is offered for the use of (15), other than the obvious fact that $r_{k}$ is to be approximated by the $k$ th power of $r^{\prime}$, and hence a function of $k$ would seem to be the most logical weighting function to use; the use of $k^{2}$ as a weighting function seemed to give the best overall fit to the series of $r_{k}$ encountered from these data. 
TABLE 2. Experimental versus theoretical slopes

\begin{tabular}{|c|c|c|c|c|c|c|c|c|c|}
\hline & $n$ & $b$ & $\beta$ & $r_{1}$ & $r^{\prime}$ & $j$ & $t_{j-2}$ & $\begin{array}{c}t(0.50 \\
j-2)\end{array}$ & $\begin{array}{c}\text { Is }|b-\boldsymbol{\beta}| \\
\text { significant } \\
\text { at } \alpha=50 \% \\
\text { level? }\end{array}$ \\
\hline \multicolumn{10}{|l|}{$\epsilon \operatorname{vs} N_{8}$} \\
\hline $\begin{array}{l}\text { Collins Data, } 8^{\circ} \\
\text { Tularosa Basin } \\
\text { Cape Canaveral }\end{array}$ & $\begin{array}{r}48 \\
161 \\
86\end{array}$ & $\begin{array}{l}0.00754 \\
.0103 \\
.01356\end{array}$ & $\begin{array}{l}0.00698 \\
.0102 \\
.00648\end{array}$ & 0.870 & 0.860 & $\begin{array}{l}(48) \\
(161) \\
6.5\end{array}$ & $\begin{array}{r}1.01 \\
.031 \\
.708\end{array}$ & $\begin{array}{l}0.68 \\
.676 \\
.73\end{array}$ & $\begin{array}{l}\text { Yes } \\
\text { No. } \\
\text { No. }\end{array}$ \\
\hline $\begin{array}{l}\Delta R_{6} \text { vs } N_{s} \\
\text { Maui Data } \\
\text { Boulder Creek Green Mountain }\end{array}$ & $\begin{array}{r}86 \\
155\end{array}$ & $\begin{array}{l}.02833 \\
.865\end{array}$ & .01610 & $\begin{array}{l}.974 \\
.944\end{array}$ & $\begin{array}{l}.950 \\
.950\end{array}$ & $\begin{array}{l}\text { 2. } 2 \\
4.0\end{array}$ & $\begin{array}{r}3.56 \\
.32\end{array}$ & $\begin{array}{l}7.6 \\
.82\end{array}$ & $\begin{array}{l}\text { No. } \\
\text { No. }\end{array}$ \\
\hline $\begin{array}{l}\Delta(\Delta R) \text { vs } N_{s} \\
\text { Boulder Creek Green Mountain ... }\end{array}$ & 86 & -.344 & -.381 & .957 & .946 & 2.4 & .60 & 2. 0 & No \\
\hline
\end{tabular}

Table 2 above shows the results of the significance tests on the slopes of the various experimental and theoretical (predicted) regression lines. The number of pieces of data is shown in the first column, the observed slope $b$ and theoretical slope $\beta$ in the second and third columns, the autocorrelation coefficient for lag of one time unit in the fourth column, and the weighted mean $r^{\prime}$ as defined in (15) in the fifth column. In column 6 the effective number of independent pieces of data, $j$, is shown, while in column 7 the value of $t_{j-2}$ is shown for the difference between $b$ and $\beta$. The next column shows the value of $t_{j-2,0.5}$, the value for the 50 percent significance level for $j-2 \mathrm{deg}$ of freedom.

Only one of the $t$ values turns out to be significant at the 50 percent level, which means that there was a better-than-even chance that such differences would have occurred by chance in the other cases. In the case of the Collins data at $\theta_{0} \cong 8^{\circ}$, the value of $t=1.01$ would not be significant at the 25 percent level; the value $t_{46}=1.01$ corresponds to $\alpha=0.34$, or a 34 percent chance that the observed deviation $|b-\beta|$ is of a random nature, and thus not significant.

From the point of view of a statistician, the results of these tests are such that no significance can be attached to any of the apparent discrepancies between theory and observation, and given reason to believe that the values of $\beta$ are theoretically sound, one could say that the results are significantly positive in nature.

The significance of the differences between the predicted and observed slopes of the regression lines for $\theta_{0}=20 \mathrm{mr}$ for the independent data check of part 4 of this paper were tested using the same method as the preceding tests, except that the 6 observations in each case were assumed to be independent. The results are summarized in table 3 and confirm the general use of the standard sample for $\theta_{0}>20 \mathrm{mr}$.

From the experimental data which are available at the present time it may be concluded that:

(1) Radio range and elevation angle errors can be predicted from the surface value of the radio refractive index, and the accuracy obtained will be generally commensurate with the estimates of residual errors made from theoretical ray-tracing considerations.
(2) The functional dependence of either angular refraction or range errors on the surface value of the refractive index as derived from the CRPL Standard $N$-profile Sample may be applied to arbitrary locations or climates without a noticeable decrease in accuracy over that obtained with a sample from the location under consideration.

(3) The effects of horizontal inhomogeneities of the refractive index, which certainly must have been prevalent over the transmission paths for which experimental data has been presented, do not appear to introduce any bias or additional residual variance into the values of observed refraction variables over those predicted from surface observations.

TABLE 3.-Comparison of slopes for independent check. Predicted slope at $\theta_{\mathrm{o}}=20 \mathrm{mr}: 0.0358 \mathrm{mr} / \mathrm{N}_{\mathrm{s}}$

\begin{tabular}{|c|c|c|c|c|c|c|}
\hline Station & $\begin{array}{l}\text { Observed } \\
\text { slope }\end{array}$ & $\begin{array}{c}\text { Dif- } \\
\text { ference } \\
b-\boldsymbol{\beta}_{0}\end{array}$ & $\sqrt{\Sigma\left(N_{s}-\bar{N}_{s}\right)^{2}}$ & $t$ & $100 \alpha$ & Significance \\
\hline $\begin{array}{l}\text { Amundsen- } \\
\text { Scott } \\
\text { Dakar } \\
\text { Bangui } \\
\text { Moscow }\end{array}$ & $\begin{array}{r}0.0520 \\
.0316 \\
.0457 \\
.0390\end{array}$ & $\begin{array}{r}+0.0162 \\
-.0042 \\
+.0099 \\
+.0032\end{array}$ & $\begin{array}{l}37.9 \\
56.5 \\
47.2 \\
44.3\end{array}$ & $\begin{array}{r}10.2 \\
.72 \\
1.37 \\
1.18\end{array}$ & $\begin{array}{l}\% \\
<0.1 \\
52 \\
25 \\
31\end{array}$ & $\begin{array}{l}\text { Very high. } \\
\text { None. } \\
\text { Very low. } \\
\text { Very low. }\end{array}$ \\
\hline
\end{tabular}

The authors thank W. L. Anderson, N. J. Beyers, R. J. Rainey, and M. C. Thompson, Jr., for making their experimental data available for this analysis.

\section{References}

Anderson, W. L., N. J. Beyers, and R. J. Rainey (Sept. 1960), Comparison of experimental and computed refraction, IRE Trans. on Antennas and Propagation AP-8, 456-461.

Anway, A. C. (June 1961), Empirical determination of total atmospheric refraction at centimeter wavelengths by radiometric means, Collins Research Report No. CRR-242S, Collins Radio Company, Cedar Rapids, Iowa.

Bean, B. R., and B. A. Cahoon (Nov. 1957), The use of surface weather observations to predict the total atmospheric bending of radio waves at small elevation angles, Proc. IRE, 45, $1545-1546$

Bean, B. R., B. A. Cahoon, and G. D. Thayer (Mar. 16, 1960), Tables for the statistical prediction of radio ray bending and elevation angle errors using surface values of the refrac- 
tive index, NBS TN44, available at the Office of Technical Services, Washington, D.C., Price 50 cents.

Bean, B. R., and G. D. Thayer (May 1959a), Models of the atmospheric radio refractive index, Proc. IRE, 47, No. 5, $740-755$.

Bean, B. R., and G. D. Thayer (Oct. 1959b), C.R.P.L. exponential reference atmosphere, NBS Mono. No. 4. Available at the Office of Technical Services, Washington, D.C. Price 45 cents.

Bennett, C. A., and N. L. Franklin (1954), Statistical Analysis in Chemistry and the Chemical Industry, John Wiley and Sons, Inc., New York, N.Y.

Brooks, C. E. P., and N. Carruthers (1953), Handbook of Statistical Methods in Meteorology, Her Majesty's Stationary Office.

Misme, P., B. R. Bean, and G. D. Thayer (Aug. 1960), Models of the atmospheric radio refractive index, Proc. IRE, 48, No. 8, 1499-1501.
Norton, K. A., J. W. Herbstreit, H. B. Janes, K. O. Hornberg, C. F. Peterson, A. F. Barghausen, W. E. Johnson, P. I. Wells, M. C. Thompson, Jr., M. J. Vetter, and A. W. Kirkpatrick (Nov. 1961), An experimental study of phase variations in line-of-sight microwave transmissions, NBS Mono. No. 33. For sale by the Superintendent of Documents, U.S. Government Printing Office, Washington, D.C. Price 55 cents.

Schulkin, M. (May 1952), Average radio ray refraction in the lower atmosphere, Proc. IRE, 40, No. 5, 554-561.

Smart, W. M. (1936), Spherical Astronomy, Cambridge University Press.

Thompson, M. C. Jr. (1962), private communication.

Wilkerson, Robert E. (1962), Divergence of radio rays by the troposphere, J. Res. NBS, 66D, (Radio Prop.) No. 4, $479-485$.

(Paper 67D3-262) 